\title{
International gamma-ray project favoured for ESA launch in 2001
}

London. A new gamma-ray observatory satellite to be launched in 2001 seems likely to become the first joint space mission between European, Russian and US space agencies. The satellite would cost an estimated $£ 400$ million (US\$600 million) and would be between ten and 50 times more powerful than the two gamma-ray observatories now in orbit.

As currently planned - and providing formal approval is obtained from each of the three space agencies - the project, known as INTEGRAL (for International Gamma-Ray Astrophysics Laboratory) would be run by the European Space Agency (ESA) as the second 'mediumsized' project in its science programme, Horizon 2000. One of the two main instruments, a germanium spectrometer, would be provided by the US National Aeronautics and Space Administration (NASA), which has also been asked to make available data-receiving facilities at two ground stations. The satellite would be launched by a Russian Proton rocket from the Baikonur Cosmodrome in Kazakhstan, in return for which scientists from the Russian Space Research Institute (IKI) would have access to the data.

INTEGRAL was given top ranking over three rival projects last week at a meeting in Paris of ESA's space science advisory committee. The choice followed a two-day meeting at the headquarters of the United Nations Educational and Scientific Organization (UNESCO), in which details of the four projects were presented to 250 space scientists.

In addition to the germanium spectrometer, INTEGRAL will carry a caesium iodide imager and two smaller instruments, an optical transient camera and an X-ray monitor. The overall goal is to improve both the angular and spectral resolution of gamma-ray observations. These will be made primarily in the plane of the galaxy, although observations will also be made of gamma-ray sources from outside the galaxy. INTEGRAL will follow up observations from the Russian GRANAT mission (which uses the French gamma-ray telescope SIGMA) launched in 1989 and now coming to the end of its mission, and the US Compton Gamma Ray Observatory, which was launched by the space shuttle in April 1991.

The advisory committee's recommendation now goes to the ESA's science programme committee, representing the scientific communities of its 13 member states, which meets in early June. The committee must also take into account the project's

international and financial implications.

INTEGRAL appears to score highly in all three areas. The international cooperation is seen as a way to reduce costs. The US contribution of $\$ 70$ million is likely to come from a NASA programme to support international collaboration, and the overall cost to the European agency is estimated to be within the budget of $£ 260$ million.

ESA's medium-sized missions are intended to complement the four larger missions that form the cornerstones of its scientific programme: the SOHO solar observatory, the XMM observatory for X-ray astronomy, a far-infrared space telescope and the cometary probe Rosetta. Thirty proposals were submitted for the 2001 launch, of which four were selected for further study.

Last week's meeting in Paris presented the results of a two-year study of the competing projects. INTEGRAL's main competition in the astronomy field is a project called PRISMA, an all-European mission to study the internal structures of stars by looking at seismic oscillations on stellar surfaces. The two other finalists are MARSNET, a scheme to set up a network of seismological and meteorological stations on the surface of MARS, and STEP, a set of instruments designed to provide

\section{Study proves Iraq used nerve gas}

Washington. An analysis of soil samples from a Kurdish village has proved for the first time that in 1988 Iraqi armed forces used nerve gas as well as mustard gas in air raids against civilians.

Physicians for Human Rights, based in Boston, and the New York group Human Rights Watch brought the samples back last summer and arranged for their analysis at the British chemical weapons laboratory at Porton Down, Wiltshire. The group says that the survival of evidence over a period of years defies expectations and improves the prospects for enforcement of the recently signed international Chemical Weapons Convention (see $\mathrm{Na}$ ture 361, 105; 1993). "This is the first time scientists have been able to prove the use of chemical weapons, and specifically nerve gas, years after the event", says Eric Stover, director of Physicians for Human Rights.

The study found a small number of parts per billion of isopropyl methylphosphonic acid (iPMPA) in soil from bomb craters in the village of Birjinni in accurate tests of the principle of equivalence between inertial and gravitational mass.

Although all four projects received high scores for their scientific content, PRISMA appears to have suffered from the high costs of its proposed European launch, and MARSNET from uncertainty over US plans for the study of the planet, while STEP was seen by some participants as requiring further technical refinements. Each will still be eligible for submission for the next 'medium-sized' mission to be launched in 2003.

British space officials do not expect any major difficulties in finding the money for INTEGRAL, because ESA science programmes are paid out of the subscriptions of member states which are calculated several years in advance. There is a similar feeling in Bonn that ESA's science programmes provide good value for money, in contrast to high-prestige projects such as the planned spaceplane Hermes and participation in the US space station. The collaboration also coincides with the desire of Germany's new science and technology minister, Matthias Wissmann, to make use of "existing investments" for research.

David Dickson

northern Iraq near the Turkish border. Alastair Hay of the University of Leeds, who chairs the group's chemical weapons group, says that iPMPA is a "unique fingerprint" of the nerve gas GB, often known as Sarin. The air attack occurred in August 1988 during a campaign against the Kurdish separatist movement.

Hay says that Sarin would normally degrade in an open environment in a matter of hours. There are no published studies on the survivability of its degradation products, although some classified work may have been carried out.

The human-rights group hopes that the findings will discourage the proliferation of chemical weapons because of fear that their use will be discovered. "The message for those involved in chemical warfare is that they might be detected, even years afterwards", says Hay. However, as many as two dozen countries are still thought to be developing chemical weapons, and the treaty will not come into force until 1995 at the earliest.

Colin Macilwain 